\title{
Semiconductor Nanolasers
}

\author{
D. Saxena, S. Mokkapati, and C. Jagadish \\ (Invited Paper) \\ Department of Electronic Materials Engineering, Research School of Physics and Engineering, \\ Australian National University, Canberra, ACT 0200, Australia \\ DOI: 10.1109/JPHOT.2012.2189201 \\ 1943-0655/\$31.00 @2012 IEEE
}

\begin{abstract}
Manuscript received February 6, 2012; accepted February 19, 2012. Date of current version April 20, 2012. This work was supported by the Australian Research Council. Corresponding author: D. Saxena (e-mail: dhruv.saxena@anu.edu.au).
\end{abstract}

\begin{abstract}
Recent progress in the field of semiconductor nanolasers is discussed. New designs have emerged that eliminate the need for a conventional Fabry-Perot cavity, bringing down the physical dimensions of the lasers below the diffraction limit. Semiconductor nanolasers are critical components for nanophotonics and offer possible integration with Si nanoelectronics.
\end{abstract}

Index Terms: Semiconductor nanowire lasers, nanolasers, plasmonic lasers.

The current electronic circuits/devices have reached their ultimate speeds, as they are only limited by inherent resistance-capacitive $(\mathrm{RC})$ delay times. Photonic devices can overcome these limitations. However, photonic circuits/devices need to shrink in dimensions to the nanoscale to be compatible with current day electronic devices. Photonic devices that offer the possibility of integration with $\mathrm{Si}$ electronics are especially interesting as these devices would be compatible with current electronics industry practices and would enable us to reap the benefits of both electronics and photonics. A laser is an inherent part of any photonic circuit, and this paper describes the recent progress in the field of semiconductor nanolasers.

Defect free direct bangdap semiconductor nanowires can be grown on Si substrates, unlike planar epitaxial layers, in spite of lattice mismatch between the substrate and the nanowire material. These nanowires are efficient light emitters and form interesting candidates for integration of efficient light emitters with Si technology. In addition, semiconductor nanowires offer tiny footprints and scalability, which has great potential for future high density optoelectronics. Semiconductor nanowire lasers have subwavelength diameters and are typically only a few micrometers long. The nanowire behaves like a Fabry-Perot cavity, with gain provided by the direct band gap semiconductor material. However, the guided modes in a nanowire are evanescent in nature and become less confined as nanowire diameter is reduced. This imposes severe size restrictions for a nanowire laser in order to attain low lasing thresholds.

The laser size can be further reduced by replacing the semiconductor Fabry-Perot cavity with metallic cavities. Metallic cavities provide the necessary positive feedback through light confinement by excitation of surface plasmon polaritons (SPPs). SPPs confine light in regions much smaller than the wavelength of light and thus bring down the laser sizes below the diffraction limit. Recently, a new concept, i.e., "lasing Surface Plasmon Amplification by Stimulated Emission of Radiation (SPASER)," was proposed [1], which uses amplification of localized surface plasmons (LSPS) to create a nanolaser. We will discuss the recent progress in semiconductor nanowire lasers, metal cavity nanolasers, and SPASERs in further detail. 

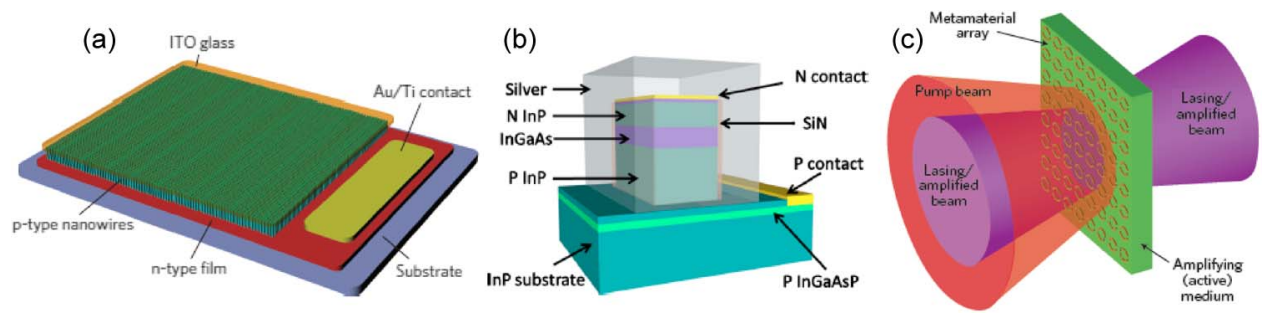

Fig. 1. (a) Schematic of electrically pumped nanowire laser device, with p-type ZnO nanowires sandwiched between ITO top contact and n-type ZnO film. Adapted by permission from Macmillan Publishers Ltd.: Nature Nanotechnology [10] (c) 2011. (b) Schematic of the metal cavity semiconductor nanolaser operating in continuous wave mode at $260 \mathrm{~K}$. Device size is $1.1 \mu \mathrm{m}$ wide $\times 2.15 \mu \mathrm{m}$ long $\times 1.55 \mu \mathrm{m}$ high. Reprinted with permission from [16] () 2011, American Institute of Physics. (c) Proposed design for a lasing SPASER which uses a metamaterial array. Adapted by permission from Macmillan Publishers Ltd.: Nature Materials [21] (c) 2010.

\section{Nanowire Lasers}

Nanowires can be grown from a variety of semiconductor materials and in a number of different structures, such as core only, core-shell, radial heterostructures, and axial superlattice structures [2]. In the last decade, lasing from nanowires of various materials, such as nitrides [3]-[5], II-VI compounds [6]-[10], III-V compounds [11], and ternary alloys [12], [13], has been reported. The majority of these demonstrations have achieved lasing by optical pumping at low temperatures. To achieve room-temperature lasing with electrical injection of carriers is a major challenge.

The first demonstration of electrically driven nanowire lasing at room temperature was in single CdS nanowires [7]. In the setup, n-type CdS nanowires were laid side-on upon a heavily doped p-type silicon substrate and Ti/Au was deposited over the nanowire with an $\mathrm{Al}_{2} \mathrm{O}_{3}$ barrier in between for electrical insulation. Electroluminescence from the nanowire end was observed at room temperature and superlinear increase in intensity was observed for injection current values above $200 \mu \mathrm{A}$.

A more recent demonstration of electrically pumped room-temperature lasing was in $\mathrm{ZnO}$ nanowires [10]. Although room temperature lasing in $\mathrm{ZnO}$ nanowires had been shown several years ago [6], electrical pumping was difficult to achieve due to issues with controllable p-type doping. In this paper, in Fig. 1(a), ZnO nanowires doped with $\mathrm{Sb}$ were grown on n-type $\mathrm{ZnO}$ thin film. Electrical contact to the nanowires was accomplished by embedding the nanowires in a thin layer of polymer and then depositing indium tin oxide (ITO) on top. The bottom contact was made by depositing $\mathrm{Au} / \mathrm{Ti}$ on the n-type $\mathrm{ZnO}$ thin film. Fabry-Perot cavity modes were observed from the $\mathrm{ZnO}$ nanowires in both photoluminescence and electroluminescence experiments. The threshold current for lasing in this device was $\sim 48 \mathrm{~mA}$. This is promising for the development of practical ultraviolet nanolasers, though further work is necessary to realize electrically pumped single nanowire lasers.

The recent demonstration of room temperature lasing in InGaAs/GaAs core-shell nanopillars ${ }^{1}$ grown on $\mathrm{Si}$ [13] is a promising accomplishment toward the monolithic integration of nanolasers with Si. In this paper, the InGaAs/GaAs heterostructures were grown on silicon without the use of metal catalysts, under conditions that are compatible with complementary metal-oxide-semiconductor (CMOS) technology. Lasing was achieved by optically pumping with an external laser, and a lasing threshold of $\sim 93 \mu \mathrm{J} \mathrm{cm}^{-2}$ was measured at room temperature. The quality $\langle Q\rangle$ factor of the nanopillar cavity was estimated to be $\sim 206$. Optical losses due to high tapering in the nanopillars and leakage to the high index Si substrate resulted in such a low $Q$ factor. In such a cavity, low order Fabry-Perot modes which experience large loss cannot lase. Instead lasing was achieved for higher order whispering gallery modes, since they experience relatively lower losses. However, these modes in principle require larger diameter nanowires to propagate. Further work in growing high-quality III-V

\footnotetext{
${ }^{1}$ They were termed nanopillars because they were much wider $(\sim 600 \mathrm{~nm})$ and shorter than most nanowires.
} 
nanowires on silicon with minimum tapering is necessary to accomplish lower lasing thresholds and a smaller device footprint.

\section{Metal Cavity Nanolasers}

The dimensions of nanowire lasers described earlier are ultimately constrained by the diffraction limit. To reduce the optical mode size and laser cavity dimensions further, especially for nanolasers emitting in the infrared, metal-dielectric cavities have to be used. The metal also serves as an electrical contact and as a heat sink. This was demonstrated in [14], in which $\operatorname{lnP} / \mathrm{InGaAs}$ heterostructure nanopillars were clad with $\mathrm{Au}$, and lasing was achieved by electrical pumping at low temperatures. The effective volume of the device was smaller than the diffraction limit. However, nonradiative recombinations, in particular Auger recombination, as a result of using Au limited the device functionality at room temperature. Instead, Ag, which has the lowest optical losses at infrared wavelengths, was used in the next demonstration, which achieved room-temperature lasing in metal-insulator-metal waveguide [15]. However, this device only had one dimension of the order of the wavelength of light (cross section $6 \mu \mathrm{m}$ long $\times 310 \mathrm{~nm}$ wide) and required large pulsed currents (peak value $\sim 6 \mathrm{~mA}$ ) for room-temperature lasing.

In the most recent demonstration of electrically pumped metal cavity nanolaser, continuous-wave operation at $260 \mathrm{~K}$ was achieved [16]. The structure comprised of $n-I n P / I n G a A s / p-I n P$ rectangular pillar which was encapsulated by Ag with a thin SiN layer in between for insulation; see Fig. 1(b). The total device volume was smaller than the cubic of the operating wavelengths in vacuum and the threshold current for lasing was $\sim 620 \mu \mathrm{A}$. Room temperature lasing with optical pumping in a metal-dielectric cavity smaller than the wavelength of emission in all three dimensions was also reported in [17] for InGaAsP active region. In another example, named nanopatch laser [18], lasing at cryogenic temperatures was observed in a small patch of metal/semiconductor/metal sandwich. Again InGaAsP was used as the semiconductor gain material.

There are other possible metal cavity nanolaser structures, with optical mode volumes below the diffraction limit, which have been demonstrated recently [19], [20]. Plasmonic crystal defect nanolasers [19] are similar to photonic crystal defect lasers except, that a plasmonic crystal defect cavity, with dimensions much smaller than a photonic crystal defect cavity, is used to localize SPPs to a volume much smaller than the diffraction limit of light. In the other demonstration [20], a plasmonic cavity is formed by $\mathrm{GaN}$ nanorods on $\mathrm{Au} / \mathrm{SiO}_{2}$ substrate. Both these structures demonstrated lasing when optically pumped at low temperatures.

\section{Lasing SPASERs}

This idea of a SPASER was first proposed by Bergman and Stockman in 2003 [1]. Unlike metallic cavities that support SPPs, it is possible to excite highly confined LSPs in arrays of nanostructured metals by coupling the metal to a semiconductor gain medium [21], see Fig. 1(c). The LSPs are amplified through emission in the semiconductor and generate intense localized optical fields. Coupling the amplified surface plasmons to radiative modes results in a "lasing SPASER." The first demonstrations of a SPASER device with subwavelength confinement of light in all three dimensions was in 2009, using a dye gain medium [22] and a II-VI semiconductor gain medium [23]. These semiconductors however either have low lifetimes and/or are not compatible with current optoelectronics industrial processes.

Semiconductor nanolasers have certainly opened up opportunities for chip-scale integration, as a result of small device volumes, electrical operation and possible integration with $\mathrm{Si}$. However, there are still further improvements to be made, especially in the fabrication process, in order to reduce surface damage, attain high-quality structures and high $Q$ factor nanostructures. These are important considerations for devices to work at room temperature with lower threshold current. Also, efficient coupling of light from a subwavelength size laser cavity to waveguides and plasmonic circuits on $\mathrm{Si}$ has yet to be demonstrated. Further work is also required to demonstrate lasing SPASERS with III-V semiconductor gain materials. 


\section{References}

[1] D. J. Bergman and M. I. Stockman, "Surface plasmon amplification by stimulated emission of radiation: Quantum generation of coherent surface plasmons in nanosystems," Phys. Rev. Lett., vol. 90, no. 2, p. 027402, Jan. 2003.

[2] O. Hayden, R. Agarwal, and W. Lu, "Semiconductor nanowire devices," Nano Today, vol. 3, no. 5/6, pp. 12-22, Oct.-Dec. 2008.

[3] J. C. Johnson, H.-J. Choi, K. P. Knutsen, R. D. Schaller, P. Yang, and R. J. Saykally, "Single gallium nitride nanowire lasers," Nat. Mater., vol. 1, no. 2, pp. 106-110, Oct. 2002.

[4] S. Gradecak, F. Qian, Y. Li, H.-G. Park, and C. M. Lieber, "GaN nanowire lasers with low lasing thresholds," Appl. Phys. Lett., vol. 87, no. 17, pp. 173111-1-173111-3, Oct. 2005.

[5] J. Heo, "Monolithic single GaN nanowire laser with photonic crystal microcavity on silicon," Appl. Phys. Lett., vol. 98, no. 2, pp. 021110-1-021110-3, Jan. 2011.

[6] M. H. Huang, S. Mao, H. Feick, H. Yan, Y. Wu, H. Kind, E. Weber, R. Russo, and P. Yang, "Room-temperature ultraviolet nanowire nanolasers," Science, vol. 292, no. 5523, pp. 1897-1899, Jun. 8, 2001.

[7] X. Duan, Y. Huang, R. Agarwal, and C. M. Lieber, "Single-nanowire electrically driven lasers," Nature, vol. 421, no. 6920, pp. 241-245, Jan. 2003.

[8] M. A. Zimmler, J. Bao, F. Capasso, S. Muller, and C. Ronning, "Laser action in nanowires: Observation of the transition from amplified spontaneous emission to laser oscillation," Appl. Phys. Lett., vol. 93, no. 5, pp. 051101-1-051101-3, Aug. 2008.

[9] Y. Xiao, C. Meng, P. Wang, Y. Ye, H. Yu, S. Wang, F. Gu, L. Dai, and L. Tong, "Single-nanowire single-mode laser," Nano Lett., vol. 11, no. 3, pp. 1122-1126, Mar. 2011.

[10] S. Chu, G. Wang, W. Zhou, Y. Lin, L. Chernyak, J. Zhao, J. Kong, L. Li, J. Ren, and J. Liu, "Electrically pumped waveguide lasing from ZnO nanowires," Nat. Nanotechnol., vol. 6, no. 8, pp. 506-510, Jul. 2011.

[11] B. Hua, J. Motohisa, Y. Kobayashi, S. Hara, and T. Fukui, "Single GaAs/GaAsP coaxial core-shell nanowire lasers," Nano Lett., vol. 9, no. 1, pp. 112-116, Jan. 2009.

[12] A. Pan, W. Zhou, E. S. P. Leong, R. Liu, A. H. Chin, B. Zou, and C. Z. Ning, "Continuous alloy-composition spatial grading and superbroad wavelength-tunable nanowire lasers on a single chip," Nano Lett., vol. 9, no. 2, pp. 784-788, Feb. 2009.

[13] R. Chen, T.-T. D. Tran, K. W. Ng, W. S. Ko, L. C. Chuang, F. G. Sedgwick, and C. Chang-Hasnain, "Nanolasers grown on silicon," Nat. Photon., vol. 5, no. 3, pp. 170-175, 2011.

[14] M. T. Hill, Y.-S. Oei, B. Smalbrugge, Y. Zhu, T. de Vries, P. J. van Veldhoven, F. W. M. van Otten, T. J. Eijkemans, J. P. Turkiewicz, H. de Waardt, E. J. Geluk, S.-H. Kwon, Y.-H. Lee, R. Notzel, and M. K. Smit, "Lasing in metalliccoated nanocavities," Nat. Photon., vol. 1, no. 10, pp. 589-594, 2007.

[15] M. T. Hill, M. Marell, E. S. P. Leong, B. Smalbrugge, Y. Zhu, M. Sun, P. J. van Veldhoven, E. J. Geluk, F. Karouta, Y.-S. Oei, R. Nötzel, C.-Z. Ning, and M. K. Smit, "Lasing in metal-insulator-metal sub-wavelength plasmonic waveguides," Opt. Exp., vol. 17, no. 13, pp. 11107-11112, Jun. 2009.

[16] K. Ding, Z. Liu, L. Yin, H. Wang, R. Liu, M. T. Hill, M. J. H. Marell, P. J. van Veldhoven, R. Notzel, and C. Z. Ning, "Electrical injection, continuous wave operation of subwavelength-metallic-cavity lasers at $260 \mathrm{~K}$," Appl. Phys. Lett., vol. 98, no. 23, pp. 231108-1-231108-3, Jun. 2011.

[17] M. P. Nezhad, A. Simic, O. Bondarenko, B. Slutsky, A. Mizrahi, L. Feng, V. Lomakin, and Y. Fainman, "Room-temperature subwavelength metallo-dielectric lasers," Nat. Photon., vol. 4, no. 6, pp. 395-399, Jun. 2010.

[18] K. Yu, A. Lakhani, and M. C. Wu, "Subwavelength metal-optic semiconductor nanopatch lasers," Opt. Exp., vol. 18, no. 9, pp. 8790-8799, Apr. 2010.

[19] A. M. Lakhani, M.-K. Kim, E. K. Lau, and M. C. Wu, "Plasmonic crystal defect nanolaser," Opt. Exp., vol. 19, no. 19, pp. 18237-18245, Sep. 2011.

[20] C.-Y. Wu, C.-T. Kuo, C.-Y. Wang, C.-L. He, M.-H. Lin, H. Ahn, and S. Gwo, "Plasmonic green nanolaser based on a metal-oxide-semiconductor structure," Nano Lett., vol. 11, no. 10, pp. 4256-4260, Oct. 2011.

[21] B. Luk'yanchuk, N. I. Zheludev, S. A. Maier, N. J. Halas, P. Nordlander, H. Giessen, and C. T. Chong, "The Fano resonance in plasmonic nanostructures and metamaterials," Nat. Mater., vol. 9, no. 9, pp. 707-715, 2010.

[22] M. A. Noginov, G. Zhu, A. M. Belgrave, R. Bakker, V. M. Shalaev, E. E. Narimanov, S. Stout, E. Herz, T. Suteewong, and U. Wiesner, "Demonstration of a spaser-based nanolaser," Nature, vol. 460, no. 7259, pp. 1110-1112, Aug. 2009.

[23] R. F. Oulton, V. J. Sorger, T. Zentgraf, R.-M. Ma, C. Gladden, L. Dai, G. Bartal, and X. Zhang, "Plasmon lasers at deep subwavelength scale," Nature, vol. 461, no. 7264, pp. 629-632, Oct. 2009. 